\title{
ФИЗИКА
}

УДК $535.4 ; 535.317$

\section{BALLISTIC AUTO-CORRELATION INTERFEROMETRY}

\author{
P. Vacas-Jacques, V. Ryabukho***, M. Strojnik, V. Tuchin ${ }^{* * *}$, G. Paez \\ Centro de Investigaciones en Optica, 37000, Leon, Guanajuato, Mexico \\ E-mail: pvj@cio.mx \\ * Saratov State University, Russia \\ E-mail: rpp@sgu.ru \\ ** Institute of Precision Mechanics and Control of the Russian Academy of Sciences, Saratov, Russia
}

We propose the implementation of a ballistic auto-correlation interferometer (BAl) for the assessment of biological tissues. We develop the theory of the interferometric technique, and demonstrate that a method to isolate ballistic from forward-scattered contributions is of central importance. We, therefore, propose theoretically and experimentally a non-linear grating-based angular filter. A monochromatic source incident on a ruled grating, positioned at grazing diffraction, is followed by a narrow slit to conform the proposed system. We validate the angular amplification experimentally, with values ranging on the order of $10-20 \mathrm{X}$. In addition, similar values of transversal beam size reduction, provide an efficient $\sim 100 X$ filtering scheme. As a preliminary study for the implementation of the BAl, we compare two transillumination schemes, which include and exclude the grating-based angular filter. Our preliminary results are encouraging, and indicate that the filter effectively isolates the information needed in the BAI.

Key words: interferometry, ballistic transillumination, angular filter, grazing diffraction, tissue imaging, tissue characterization.

\section{Баллистическая автокорреляционная интерферометрия}

П. Вакас-Жакс, В. Рябухо, М. Стройник, В. Тучин, Г. Паез

Рассматривается применение баллистической автокорреляционной интерферометрии (БАИ) для контроля параметров биологических тканей. Представлена теория интерференционного эксперимента и показано, что автокорреляционный метод интерферометрии позволяет изолировать и выделить в рассеянном вперед излучении нерассеянное, баллистическое, излучение. Для реализации метода предложено использовать нелинейный дифракционный угловой фильтр, действие которого основано на пространственной фильтрации света с помощью узкой щели света в скользящем дифракционном порядке. Реализованное с помощью такого фильтра угловое увеличение составило 10-20X. Такое же увеличение дает поперечное масштабирование дифрагированного пучка света, что в целом обеспечивает эффективную 100X схему угловой фильтрации рассеянного излучения. Выполнено сравнение двух схем БАИ с угловым фильтром и без его использования. Экспериментальные результаты подтверждают теоретические положения и показывают эффективное действие фильтра для реализации меТода БАИ.

Ключевые слова: интерферометрия, баллистическое просвечивание, угловой фильтр, дифракция, имиджинг биологических тканей, диагностика ткани.

\section{Introduction}

Transmission of light in a turbid medium, such as tissue, is governed by the radiative transport equation $[1-4]$. The attenuation characteristics of the media are required to describe the propagation of radiation [5]. More specifically, the necessary parameters are the absorption coefficient $\mu_{\mathrm{a}}\left[\mathrm{mm}^{1}\right]$, the scattering coefficient $\mu_{\mathrm{s}}\left[\mathrm{mm}^{-1}\right]$, and the anisotropy factor $g$. The first two coefficients describe the probability of absorption and scattering of light per unit length. Meanwhile, the third parameter determines the average direction of propagation after interaction. In addition, the anisotropy of propa-

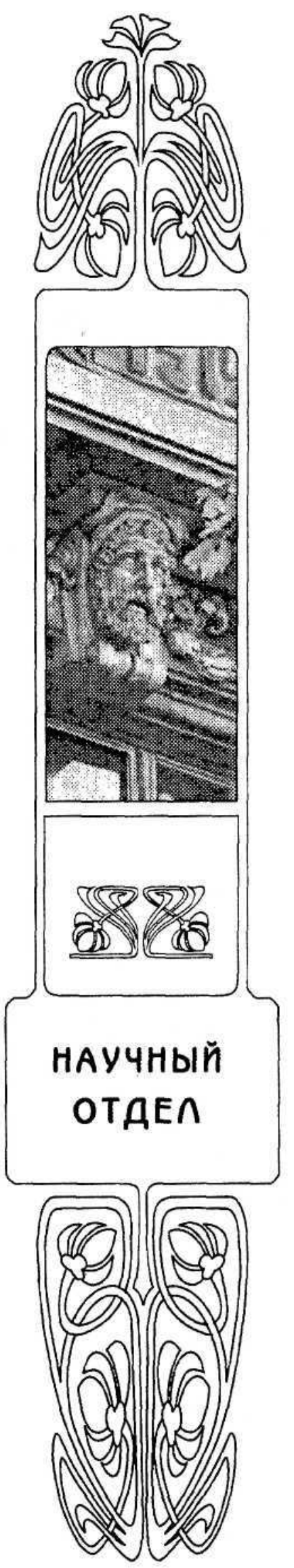


gation after interaction. In addition, the anisotropy factor is related to a specific phase function, which accounts for the pattern of scattering distinctive of the turbid medium. Biological tissues commonly exhibit phase functions with marked forward-directed patterns [6,7]. Therefore, incident and scattered propagation directions are very similar.

One of the challenges of implementing a transillumination technique with ballistic photons resides in the rapid attenuation of such ra- diation. A perhaps more complicated endeavor however, is the separation of ballistic photons from those that have undergone scattering. The latter assertion is further validated by acknowledging that the anisotropy factor is usually greater than 0.9 for biological tissues [7]. In Fig. 1, we give an example of the intermingled presence of ballistic and scattered photons after transillumination. The sample is $10 \mathrm{~mm}$ thick; $\mu_{\mathrm{a}}=0\left[\mathrm{~mm}^{-1}\right], \mu_{\mathrm{s}}=0.591\left[\mathrm{~mm}^{-1}\right]$, and $g=0.93$.
(1)

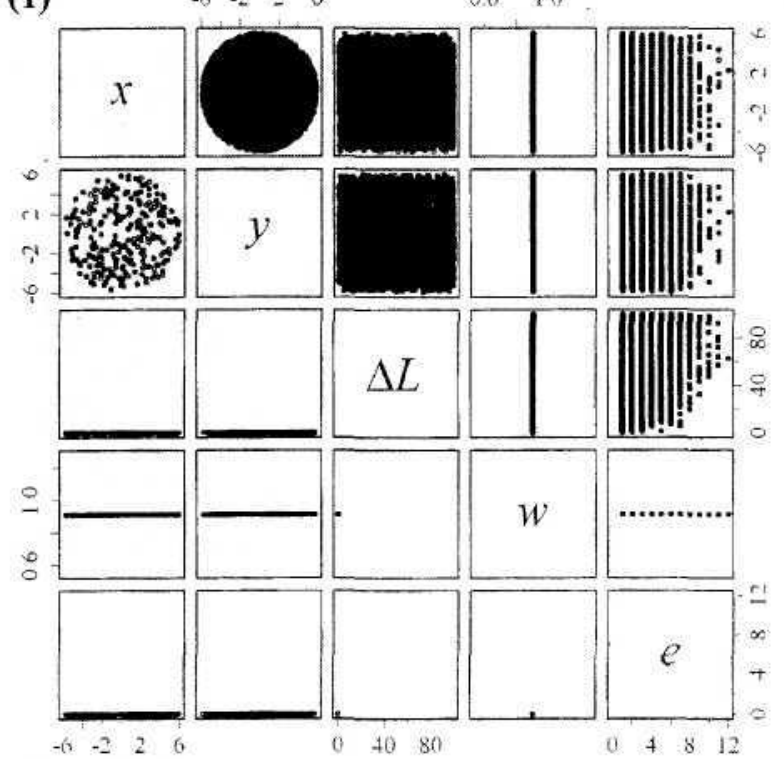

(2) $-6=20$ $06 \quad 10$

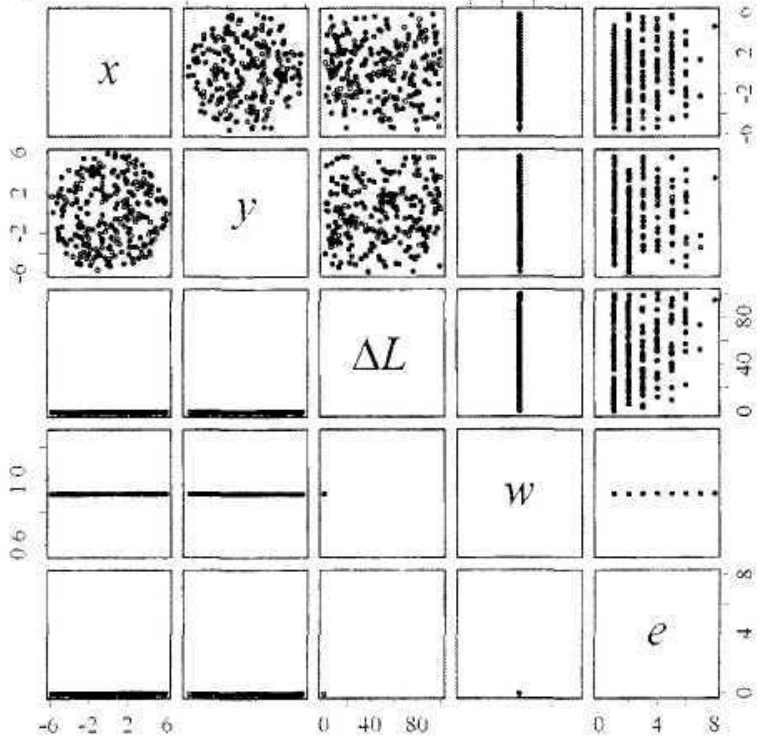

Fig. 1. Ballistic and scattered contributions are commingled after transillumination: immediate (1) and distant (2) observation

In Fig. 1, data below and above the main diagonal correspond to that of ballistic and scattered photons. Each row-column intersection is a scatter plot of the associated variables in the diagonal. $x$ and $y$ are transversal coordinates (in $[\mathrm{mm}]), \Delta L$ represents optical path-length difference (in $[\mu \mathrm{m}]$ ), $w$ is weight, and $e$ stands for the number of scattering events. As seen from Fig. 1, distinction of ballistic from scattered photons is challenging. Despite the observation position, near (1) or far (2), both contributions lie close together. Such behavior of radiation transport is relevant in the ballistic autocorrelation interferometer (BAI). In Fig. 2, we depict a schematic of the BAI.

In the following section, we present the theoretical foundation of the BAI. We emphasize the importance of adequately selecting the

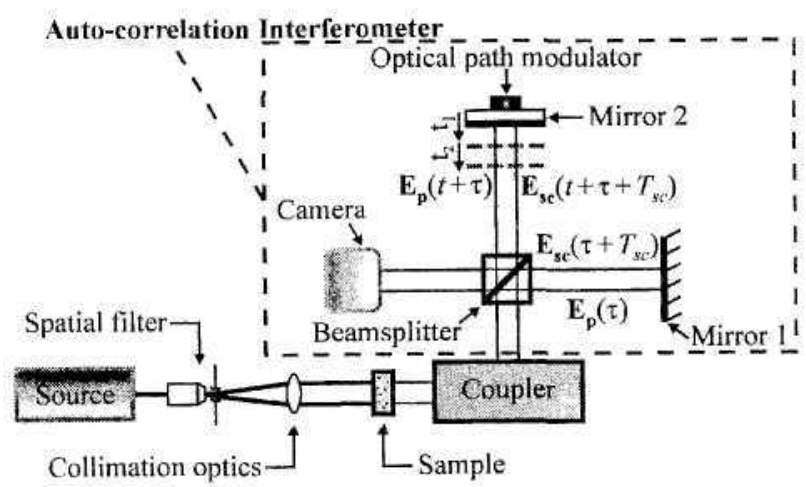

Fig. 2. In the BAI a coupler is necessary to isolate ballistic from forward-scattered radiation

information of interest. For this purpose, we suggest the implementation of a non-linear grating-based angular coupler. In the third part of this work, we describe experimental setup and 
methodology. In section four, we then assess the performance of the angular coupler. In addition, we compare two transillumination schemes, which include and exclude the grating-based system. We devote the fifth section to discuss possible improvements. Finally, we summarize the results of this work and present plausible future research directions.

\section{Theory}

As pointed out in the introduction, the radiation impinging on the sample suffers diverse interactions in the turbid medium. As a result of such interactions, the radiation is transmitted, reflected, or absorbed. In essence, we may speak of two contributions that are $t$ ransmitted: forward-scattered and ballistic. The transmitted components are especially relevant for the implementation of a ballistic auto-correlation interferometer. The coherent superposition of transmitted beams is employed in the BAI to enable characterization and imaging of turbid media. Electrical fields are associated with incident $\mathbf{E}_{\mathrm{i}}$, forward-scattered $\mathbf{E}_{\mathrm{sc}}$, and ballistic or passthrough $\mathbf{E}_{\mathbf{p}}$ contributions. At the superposition plane of the BAI, see Fig. 2, ballistic and forward-scattered components are observed:

$$
\begin{aligned}
P\left(t, T_{s c}\right) & \propto\left\langle\left\{\left[\mathbf{E}_{\mathrm{p}}(\tau)+\mathbf{E}_{\mathrm{sc}}\left(\tau+T_{s c}\right)\right]+\right.\right. \\
& \left.+\left[\mathbf{E}_{\mathrm{p}}(t+\tau)+\mathbf{E}_{\mathrm{sc}}\left(t+\tau+T_{s c}\right)\right]\right\} \times \\
& \times\left\{\mathbf{E}_{\mathbf{p}}(\tau)+\mathbf{E}_{\mathrm{sc}}\left(\tau+T_{s c}\right)\right]+ \\
& \left.\left.+\left[\mathbf{E}_{\mathrm{p}}(t+\tau)+\mathbf{E}_{\mathrm{sc}}\left(t+\tau+T_{s c}\right)\right]\right]^{*}\right\rangle .
\end{aligned}
$$

Here, $P$ stands for detected power. $\tau$ is the reference time for partial coherence modeling. $t$ represents the modulation time, and $T_{s c}$ describes

the random time that scattered photons take to traverse the sample. The chevrons denote time average and an asterisk demarcates complex conjugation. Throughout this work, we use bold letters for complex-valued quantities.

In Eq. (1), there are no reference contributions. This is one principal characteristic of the auto-correlation interferometer $[8,9]$. In addition, we ascertain that forward-scattered and passthrough contributions are commingled. We may further simplify the expression of interference by relating the ballistic field to the incident one:

$$
\begin{aligned}
\mathbf{E}_{\mathbf{p}}=\mathrm{R}_{g} & \exp \left[-\mathrm{i} \frac{2 \pi}{\lambda} 2 n_{g} d\right] \exp \left[-\frac{1}{2} \int_{0}^{D} \mu_{\mathrm{t}}(z) \mathrm{d} z\right] \times \\
& \times \exp \left[-\mathrm{i} \frac{2 \pi}{\lambda} \int_{0}^{D} n_{s}(z) \mathrm{d} z\right] \mathbf{E}_{\mathrm{i}} .
\end{aligned}
$$

Here, the Fresnel reflection coefficient is $\mathrm{P}_{g}$ and we denote the wavelength as $\lambda$. The refractive index and thickness of the container, for in-vitro studies, are $n_{g}$ and $d$. In addition, a sample of thickness $D$, attenuation coefficient $\mu_{\mathrm{t}}=\mu_{\mathrm{a}}+\mu_{\mathrm{s}}$, and refractive index $n_{s}$ is considered. In previous publications, we presented more succinctly the expression in Eq. (2) as $\mathbf{E}_{\mathrm{p}}=\gamma_{\mathrm{m}} \mathbf{E}_{\mathrm{i}}[10,11]$. Nonetheless, the complete equation serves to ascertain the rapid decay of ballistic radiation.

In contrast to the behavior of ballistic radiation, scattered contributions are random. Thus a simple expression between scattered and incident fields is not at hand. However, the fields are related to each other. Previously, we have employed the notation $\mathbf{E}_{\mathrm{sc}}=\gamma_{\mathrm{sc}} \mathbf{E}_{\mathrm{i}}$ for such purpose $[10,11]$. By relating ballistic and scattered contributions to the incident field, we obtain the partial coherence model of the BAI:

$$
\begin{aligned}
P\left(t, T_{s c}\right) & =\Gamma_{i, i}(0)\left\{\left(1-k_{s p 1}\right)\left(1-k_{s p 2}\right) \times\left(\left|\gamma_{\mathrm{m}}\right|^{2}+\left|\gamma_{\mathrm{sc}}\right|^{2}+2\left|\gamma_{\mathrm{m}}\left\|\gamma_{\mathrm{sc}}\right\| \gamma_{\mathrm{i}, \mathrm{i}}\left(T_{s c}\right)\right| \cos \left[\Phi_{i, i}\left(T_{s c}\right)\right]\right)+\right. \\
& +k_{s p 1} k_{s p 2}\left(\left|\gamma_{\mathrm{m}}\right|^{2}+\left|\gamma_{\mathrm{sc}}\right|^{2}+2\left|\gamma_{\mathrm{m}}\left\|\gamma_{\mathrm{sc}}\right\| \gamma_{i, \mathrm{i}}\left(T_{s c}\right)\right| \cos \left[\Phi_{i, i}\left(T_{s c}\right)\right]\right)+ \\
& +2 \sqrt{k_{s p 1}} \sqrt{1-k_{s p 1}} \sqrt{k_{s p 2}} \sqrt{1-k_{s p 2}} \times\left(\left.|| \gamma_{\mathrm{m}}\right|^{2}+\left|\gamma_{\mathrm{sc}}\right|^{2}\right]\left|\gamma_{\mathrm{i}, \mathrm{i}}(t)\right| \cos \left[\Phi_{t, i}(t)\right]+ \\
& \left.\left.+\left|\gamma_{\mathrm{m}}\left\|\gamma_{\mathrm{sc}}\right\| \gamma_{\mathrm{i}, \mathrm{i}}\left(T_{s c}-t\right)\right| \cos \left[\Phi_{t, i}\left(T_{s c}-t\right)\right]+\left|\gamma_{\mathrm{m}}\left\|\gamma_{\mathrm{sc}}\right\| \gamma_{i, \mathrm{i}}\left(T_{s c}+t\right)\right| \cos \left[\Phi_{i, i}\left(T_{s c}+t\right)\right]\right)\right\} .
\end{aligned}
$$

Here, $\Gamma_{i, i}(0)$ is the total collected power upon proving a small area. $k_{s p 1}$ and $k_{s p 2}$ denote splitting coefficients. In the Michelson configuration, $k_{s p 2}=1-k_{s p 1}$. Finally, $\left\{\gamma_{\mathrm{i}, \mathrm{i}}(\tau) \mid\right.$ and $\Phi_{i, l}(\tau)$ designate amplitude and phase of the complex degree of self-coherence.
From Eq. (3), we predict commingled detection of forward-scattered and ballistic photons. Therefore, we ascertain the necessity of adequately coupling the information of interest. In addition, from Fig. 1 we may identify the major competing process in the BAI. For this pur- 
pose, we analyze the weight to number of events plot ( $w$ vs $e$ ) for scattered photons. Such information is found in the fourth row and fifth column intersection. For simplicity, we examine the data of scenario (2). From the plot we establish that scattered photons with few events are the main competing process. The latter assertion is valid, because such photons maintain a propagation direction similar to that of ballistic radiation. Furthermore, the weight of such scattered radiation is significant, as opposed to that of highly diffuse contributions.

Particularly, single scattered radiation represents a diffuse contribution that is difficult to overcome. Single scattered photons, especially in highly forward-directed samples, do not suffer an important phase delay. Thus ballistic techniques which employ coherence gating, or ultra-fast imaging, encounter challenges while dealing with such radiation $[10,11]$. However, single scattered photons indisputably modify their propagation direction. Hence, a technique which exploits such angular differences could be optimal to feed the BAI. For this purpose, we suggest the use of a ruled diffraction grating as coupling device. The peculiarity of our proposal resides in the regime of operation of the optical element. The grating is positioned such that one of the first diffraction orders $m=+1$ occurs at a grazing angle, as shown next.

The behavior depicted in Fig. 3 is supported by the theory of diffraction gratings. In fact, functioning of the coupler is based on conical diffraction theory. For in-plane geometries, diffracted and incident beams are related by the grating equation $[12]$ :

$$
\sin \beta=\sin \alpha+G m \lambda .
$$

Consider in Fig. 3 that the incidence angle $\alpha$ is $13.8^{\circ}$ and that $\mathrm{He}-\mathrm{Ne} \lambda=632.8[\mathrm{~nm}]$ light is incident on the grating, which has a groove frequency $G$ of $1200[1 / \mathrm{mm}]$. Then, the first diffraction order $(m=+1)$ occurs roughly at $\beta=$ $=86^{\circ}$. By analyzing figures 1,2 , and 3 , we note that the grazing diffraction grating might be an adequate coupler for the BAI. The latter assertion is valid, provided that the diffractive element is sensitive to minute variations in incidence angles. Mathematically, this is assessed by finding the derivative of Eq. (4):

$$
\Delta \beta=\left(\frac{\cos \alpha}{\cos \beta}\right) \Delta \alpha .
$$

The importance of Eq. (5) is that angular variations in the diffracted beam are amplified nonlinearly by $(\cos \alpha / \cos \beta)$. This assertion is especially evident for near grazing diffraction geometries, $\beta \rightarrow \pi / 2$ [rad]. In fact, for the parameters of the coupler mentioned before, the amplification is $15 \mathrm{X}$. In Fig. 4, we present the behavior of scattered and ballistic radiation, before and after interaction with such coupler.

By inspection of the setup in Fig. 3, we observe a reduction of the transversal beam size. The decrease in beam size is also given by $(\cos \alpha / \cos \beta)$. For the case under consideration, such reduction is $\sim 15 \mathrm{X}$. Both angular amplification and decrease in beam width affect scattered photons. Therefore, in the BAI, the use of a narrow slit in conjunction with a grazing diffractive element may provide a filtering scheme of $225 \mathrm{X} ;(\cos \alpha / \cos \beta)^{2}$. This behavior, in turn, implies that ballistic photons may be exclusively coupled to the interferometer. Therefore, Eq. (3) simplifies accordingly.

$$
\begin{aligned}
P(t)=\mid & \left.\gamma_{\mathrm{m}}\right|^{2} \Gamma_{i, t}(0)\left\{\left(1-k_{s p 1}\right)\left(1-k_{s p 2}\right)+\right. \\
+k_{s p 1} k_{s p 2}+ & 2 \sqrt{k_{s p 1}} \sqrt{1-k_{s p 1}} \sqrt{k_{s p 2}} \sqrt{1-k_{s p 2}} \times \\
\times & \left.\left|\gamma_{\mathrm{i}, \mathrm{i}}(t)\right| \cos \left[\Phi_{i, i}(t)\right]\right\} .
\end{aligned}
$$

By means of the expression in Eq. (6), we may identify several regimes of operation for the BAI. First, tissue characterization may be rea-

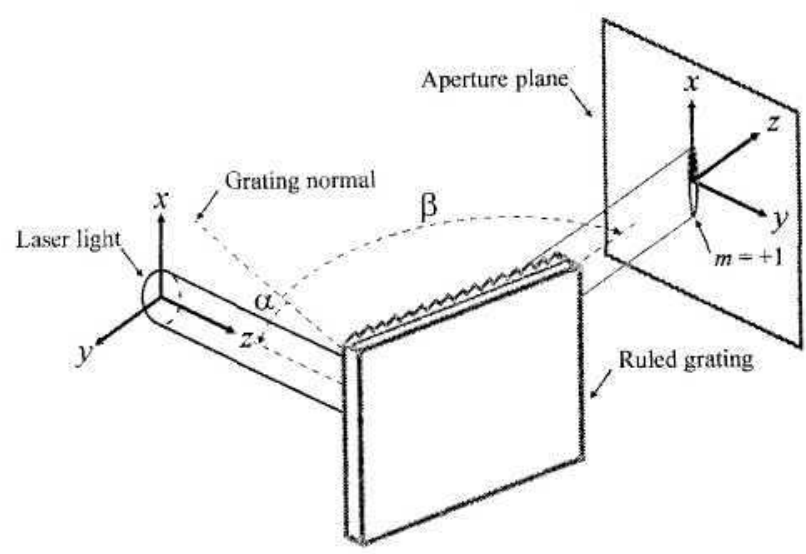

Fig. 3. A grazing diffraction element followed by a narrow slit conform the angular coupler 
(1)

$\begin{array}{llll}-6 & -2 & 2 & 6\end{array}$

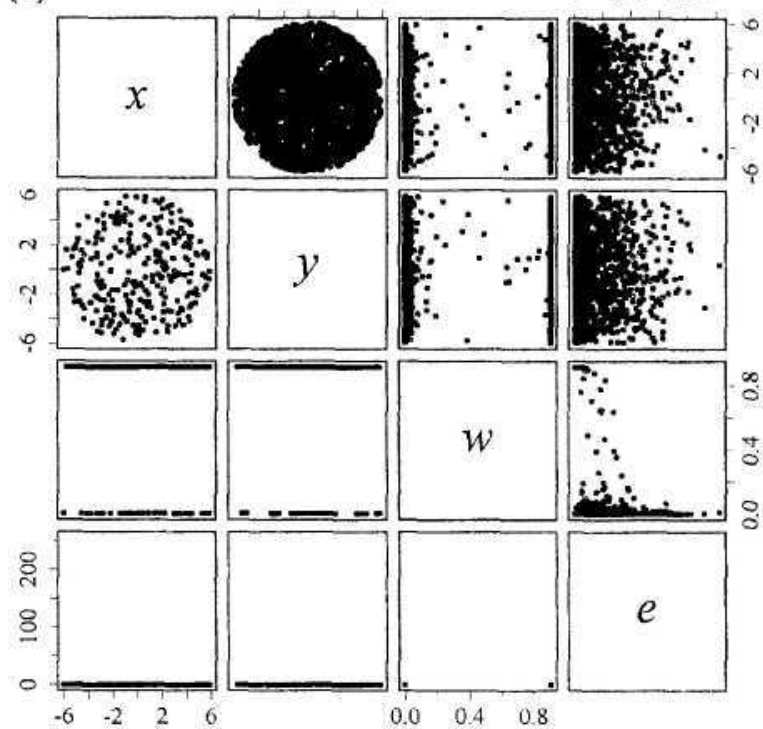

(2)

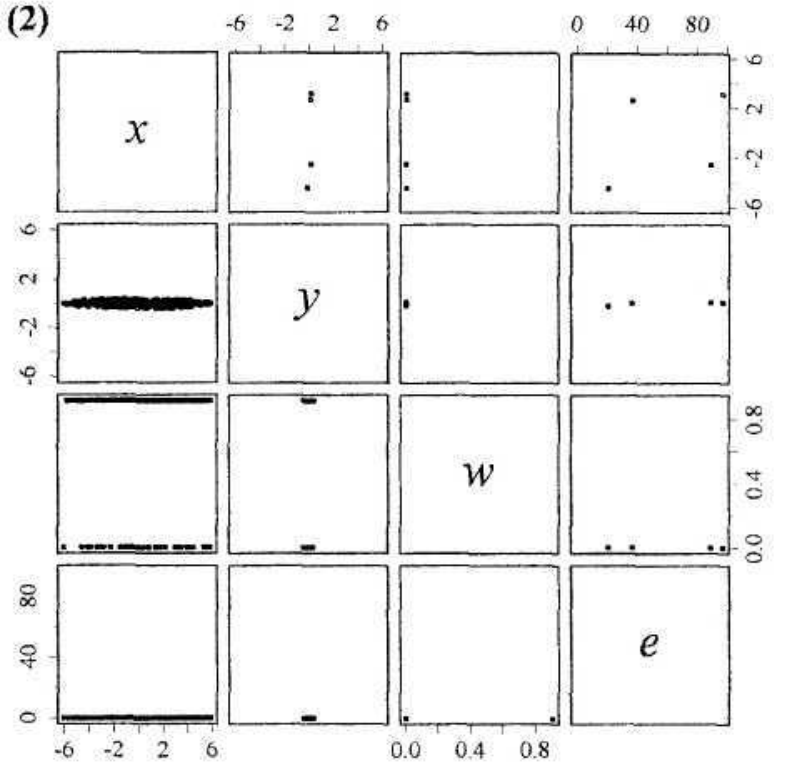

Fig. 4. Exclusion of the grazing grating (1) implies commingled presence of ballistic and scattered radiation, whereas inclusion of the $15 \mathrm{X}$ coupler (2) provides a means to overcome noise

lized by employing phase-locked techniques. In order to perform the latter, an accurate modulation reference is necessary. The information recovered by such method is proportional to $\left|\gamma_{\mathrm{m}}\right|^{2}$. In addition, a similar approach may yield the phase of the object under study. For this case, however, instead of phase-locked detection schemes, a phase-stepping approach is required. The second regime of operation corresponds to that of tissue imaging. From Eq. (6), we ascertain that pixel maxima are related to the integrated attenuation of the sample. In addition, another important aspect related to the application of tissue imaging, concerns the reduction of beam extent implicit in the configuration. Such beam reduction must be compensated according$\mathrm{ly}$, in order for the tissue imaging regime to be applicable. The prime issue to address, nonetheless, is the feasibility of implementing the grating-based angular filter.

\section{Materials and Methods}

3.1. Experimental setup. From theory, we expect a non-linear relation between diffracted and incident beams. The latter assertion is particularly relevant for the purposes of implementing a BAI. We are, therefore, interested in finding the rate of change of diffraction angle as a function of incidence angle. As a consequence, an important element of the experiment is the rotating scheme employed to vary the incidence angle, $\alpha \pm \Delta \alpha$. We utilize a $360^{\circ}$ rotation stage, which provides a $15^{\circ}$ interval with 1 arc $\min$ resolution (Precision rotary stage 7R129, Standa Optics, Lithuania).

In addition, as the coupler approaches the grazing condition, we expect the diffracted angle to increase considerably. Thus, in the setup, another important component is the optical system needed to capture such variations. The optical system we employ exhibits a variable fnumber. Furthermore, the diaphragm enables accurate constriction of the system to the paraxial regime. This regime is of importance, because accurate system alignment is facilitated. Accurate alignment is in turn required to calculate, by means of geometrical optics, the angular deviation of the diffracted beam. In Fig. 5, we depict a schematic of the experimental setup.

From Fig. 5, we recognize several important features related to the experiment. First, the mechanics of the rotating element play an important role when fine movements are in order. A mechanical malfunctioning, for instance a non-continuous movement, will manifest as a deviation from the expected performance. Second, the f-number of the optical system determines the incidence angle range that may be assessed. Thus if a considerable range is in play, it must be subdivided accordingly. Third, in order to determine the angular change after dif- 


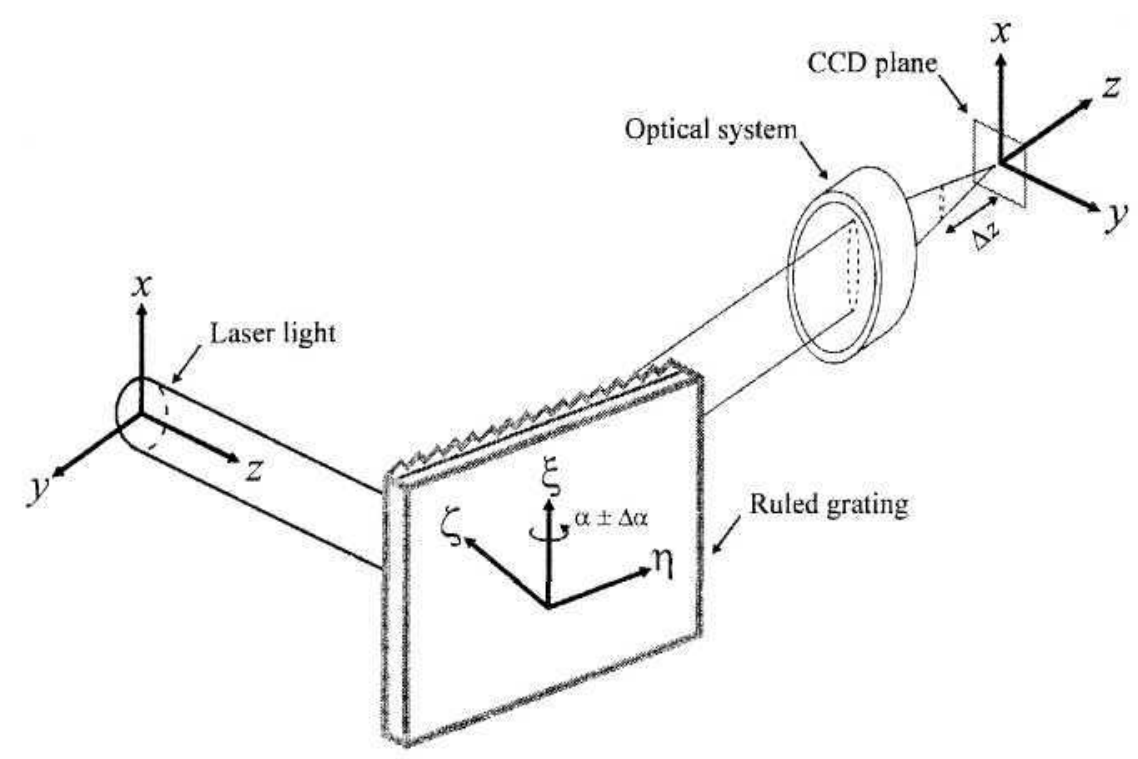

Fig. 5. Schematic representation of the system employed to validate the non-linear behavior of the grating-based angular coupler

fraction, we require the magnification of the optical system. In Fig. 6, we show a picture of the arrangement as implemented in our laboratory.

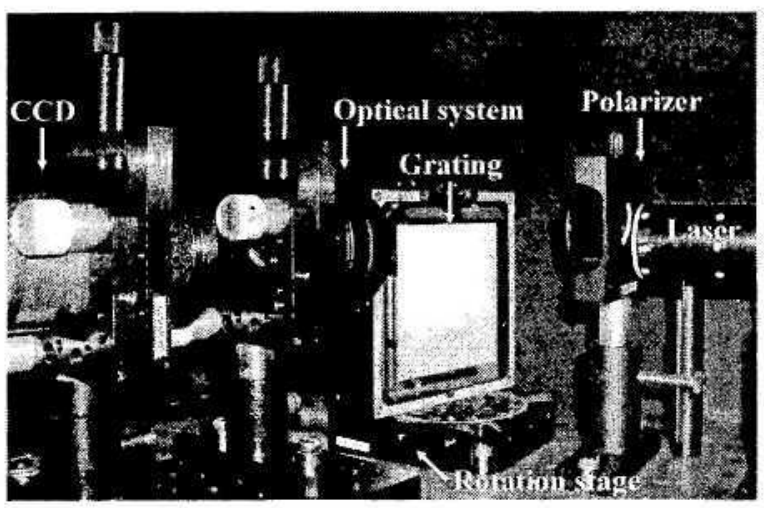

Fig. 6. Experimental setup utilized to validate the non-linear behavior of the proposed coupler

3.2. Methodology. Due to the fact that the aperture of the optical system is finite, we subdivide the range of incidence angles of interest. We select a determined angular interval and align the optical system, including the CCD. We perform the latter by working on the paraxial regime of the optical system. Once the alignment is achieved, we verify the latter by displacing the CCD (along the $z$-axis in Fig. 5), which is mounted on a translational stage. This procedure is important because the diffracted angle is calculated by using geometrical optics and trigonometry. The translational stage provides one of the parameters needed in the calculations: the transversal reference position of the CCD. Meanwhile, we obtain the other information from CCD measurements.

From Fig. 5, we observe that all diffracted beams should converge in the image plane. In terms of geometrical optics, this is readily explained because we are tracing exclusively one ray. The latter, as shown in Fig. 5, always leaves the object plane (i.e., the diffraction grating) from the same place. After determining the location of convergence, we move the CCD a determined amount, $\Delta z=3[\mathrm{~mm}]$, along the optical axis. Thereafter, we calculate the transversal displacement of the beam in the CCD plane. In Fig. 7, we show a selected set of measurements from one of the experiments.

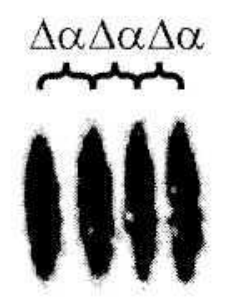

Fig. 7. Transversal displacement of diffracted beam as incidence angle varies, $\Delta \alpha=7$ [ $\operatorname{arcmin}$ ]

Depending on the angular range under consideration, we perform a sequence of measurements with an angular interval of 1 arc min. 
Thus $\Delta \alpha=1 / 60 \mathrm{e}$ in Eq. (5), which implies that the derivative may be calculated accurately. Nonetheless, in the experiment, mechanical imperfections restrict the minimum feasible range. In our case, we space the measurements from five to eleven arc min. Worth of mention is that each sequence of measurements is repeated 10 times to account for statistical uncertainties.

From the alignment procedure, we determine the reference transversal position and the movement induced along the optical axis of the CCD. In addition, from the sequential variation of the incidence angle, we calculate the corresponding transversal displacement of the diffracted beam (see Fig. 7). Thereafter, we compute the values of diffraction angles by employing trigonometry and the magnification factor of the optical system. Finally, from successive measurements, we calculate the response of the system.
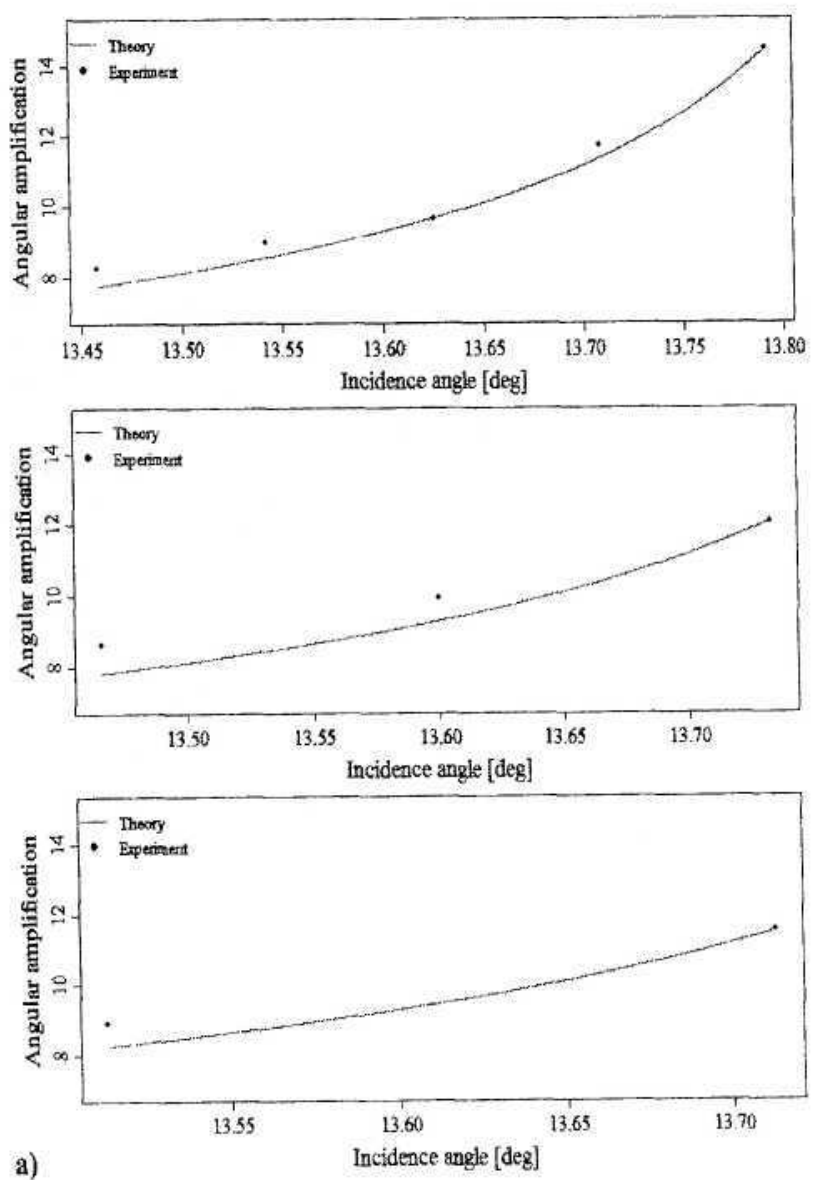

a)

$$
\text { Incidence angle [deg] }
$$

Fig. 8. Comparison of experiment with theory for two configurations: a) $8-14 \mathrm{X}$ and b) $10-22 \mathrm{X}$

\section{Results}

4.1. Non-linear grating-based angular filter. In the experiment, the exact value of the incidence angle $\alpha$ is unknown. The angular increment $\Delta \alpha$, in contrast, is under control of the experimenter. From the measurements, we determine the relative increment in diffracted angle $\Delta \beta$. This conjoint information yields the sought rate of change, $\Delta \beta / \Delta \alpha$. In addition, as shown in Eqs. (4) and (5), the diffractive configuration and the incidence angle may also be employed to calculate the rate of change. Since the incidence angle is not known, we compute the theoretical behavior from the rate of change obtained experimentally. In the theoretical calculations, we employ the greatest value of $\Delta \beta / \Delta \alpha$ as reference. From such value of the rate of change, we compute the corresponding incidence angle, and the theoretical curve for the configuration. In Fig. 8, we compare the results of the experiment with theory for two amplification schemes: a) 8-14X and b) $10-22 X$.
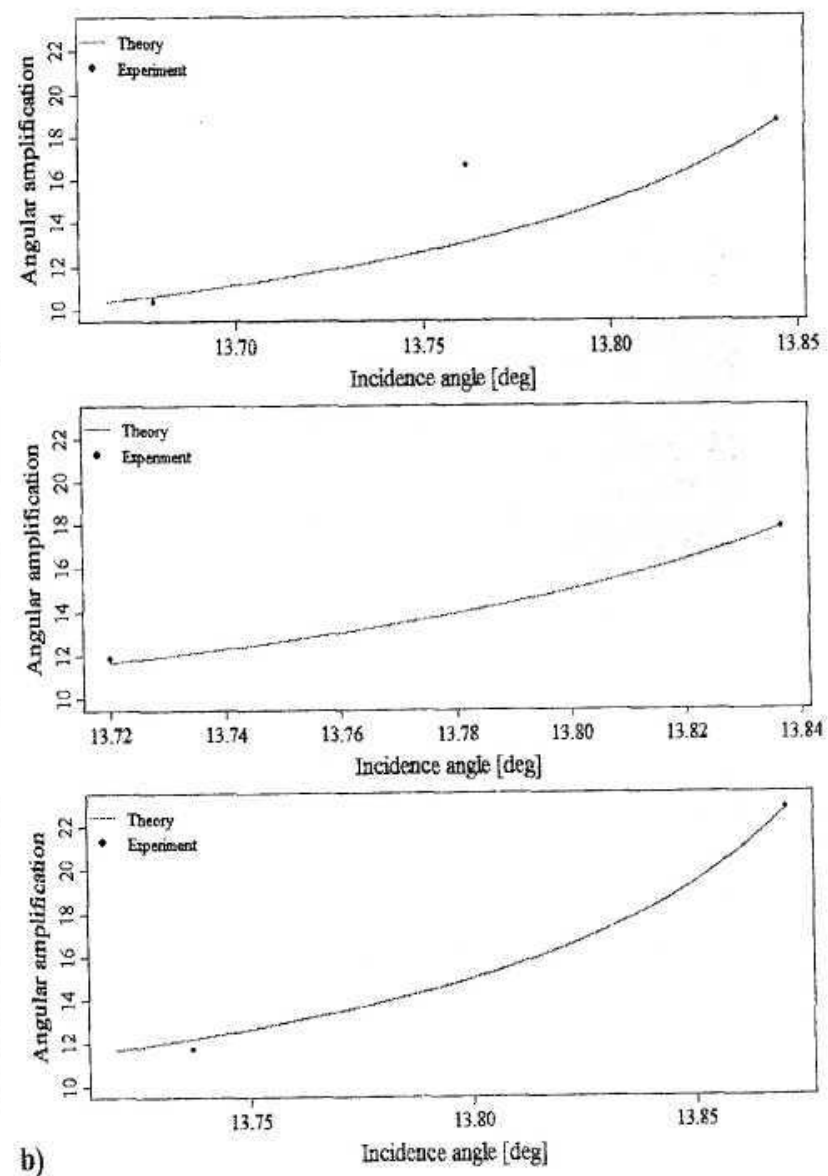
The angular amplification depicted in Fig. 8 is the rate of change of Eq. (5). From the figure, we ascertain agreement between theory and experiment. For the case of lower amplification, theoretical and experimental values exhibit good correspondence. Certain experimental values in the greater amplification scheme tend to oscillate more. These discrepancies are readily justified by acknowledging the non-linear behavior of the system.

In Fig. 8, the non-linear behavior of the system is evident. In addition, a relevant feature of the design of the experiment may be recognized from the same representation. Namely, marked non-linear response is confined to a few arc minutes. For example, if $\alpha=13.8^{\circ}$ then $\Delta \beta / \Delta \alpha \sim 15$. However, if we change the incidence angle by roughly 7.5 arc min, the grazing condition is exceeded. Due to the specifications of our equipment, the highly non-linear behavior of the filter was not assessed. Recently, in the field of spaceborne angular sensors, such regime has been explored $[13,14]$. Nonetheless, from Fig. 8, we may conclude that amplification values ranging from $10-20 \mathrm{X}$ are feasible with 1 arc min resolution mounts.

\subsection{Transillumination: traditional versus} grating coupler schemes. We now address a topic that is closely related to the BAI. In order to assess the applicability of the grating coupler, we perform two transillumination experiments. In the first scenario, we perform traditional transillumination. In Fig. 2, this experiment entails the replacement of the coupling device by a $\mathrm{CCD}$ or a camera. In the second setting, we implement the grating-based scheme. The coupler in Fig. 2 is replaced by the diffractive element at grazing regime. In addition, we employ a set of cylindrical lenses (in telescope arrangement) and a CCD to recover sample information [15].

The sample is composed of $1.5 \mu \mathrm{m}$ polystyrene latex spheres suspended in deionized water. The choice of particle size is related to the anisotropy factor, which is a central parameter in the BAl. We employ Mie theory to calculate the attenuation behavior of the sample [16]. Specifically, the anisotropy factor equals 0.935 ; a value characteristic of forward-scattering media. We employ a rather transparent sample, which is similar to the scenario encountered in dental enamel tissues [17]. The sample is held in a cuvette. Furthermore, a line label of such container is employed as the reference object. Images of reference object, traditional transillumination (employing only a CCD and a camera), and grating-based schemes are shown in Fig. 9.

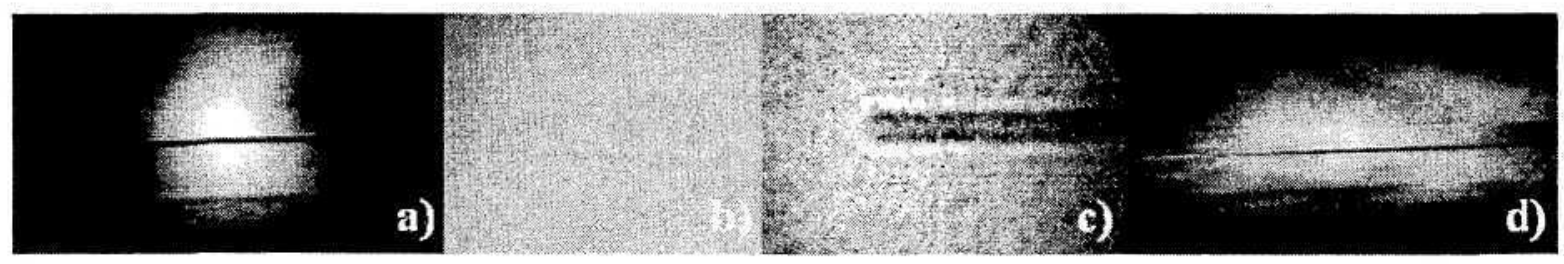

Fig. 9. Transillumination results: a) Reference, b) Traditional only CCD, c) Traditional with camera, d) Grating-based scheme

We concentrate on the salient features of the images presented in Fig. 9. First, in the traditional transillumination schemes, Figs. 9 b) and c), we observe a significant amount of forwardscattered contributions. Second, speckle patterns are also recognizable from these representations. These patterns may be more easily identified in a sequence of frames. Third, the exposition time necessary for acquiring the figures in d) is more significant than the corresponding measure for traditional transillumination. More specifically, the exposition time $t_{\exp }$ (VS-CTT-259-2001, Videoscan, Russia) is calculated from the rela- tion in Eq. (7). In Table, we present the corresponding measures for the images in Fig. 9.

$$
t_{\exp }=20000 \times T V S S+64 \times T H S S+45.2[\mu \mathrm{s}] .
$$

Exposition times for the transillumination results of Fig. 9

\begin{tabular}{|l|c|c|c|}
\hline \multicolumn{1}{|c|}{ Arrangement } & TVSS & THSS & $t_{\exp }[\mu \mathrm{s}]$ \\
\hline Traditional only CCD* & 0 & 33 & 2160 \\
\hline Traditional with camera* & 0 & 9 & 620 \\
\hline Grating-based scheme & 7 & 1 & 140110 \\
\hline
\end{tabular}

* THSS values for traditional mo dalities are approximate. 
Due to the presence of the diffractive element, the grating-based configuration exhibits an implicit reduction of power at the CCD. Despite this fact, the exposition times shown in $\mathrm{Ta}$ ble, for the grating-based scheme, are orders of magnitude greater than those of traditional transillumination. Such behavior is characteristic of the rapid decay of ballistic radiation. Therefore, these results suggest that the proposed method may be suited for coupling selectively the radiation required in the BAI.

\section{Discussion}

The device we have introduced in this work may be implemented readily for applications involving tissue characterization. In our group, we are also interested in performing tissue imaging. Furthermore, we would like to complement the

(1)

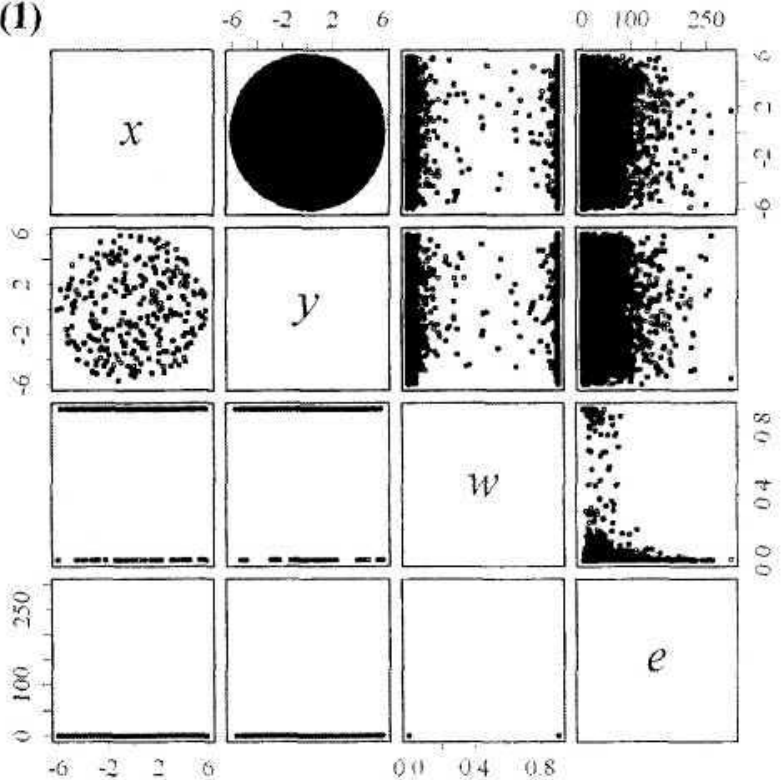

technique with interferometric methods. For imaging applications, we foresee challenges due to the marked asymmetry of the spatial distribution, see Fig. 4. In order to overcome such asymmetry, we may introduce a second diffractive element.

Taking Fig. 3 as reference, we should position the additional grating in the aperture plane. The grooves need to be parallel to the $y$ axis, and by rotating the element we should reach the grazing diffraction condition. An advantage of this configuration is that the angular amplification occurs in a transversal direction. In addition, if the characteristics of the gratings match, we anticipate that the spatial asymmetry will be removed. This behavior implies that circular diaphragms may be used to block scattered light. In Fig. 10, we depict the theoretical response of the improved coupler.

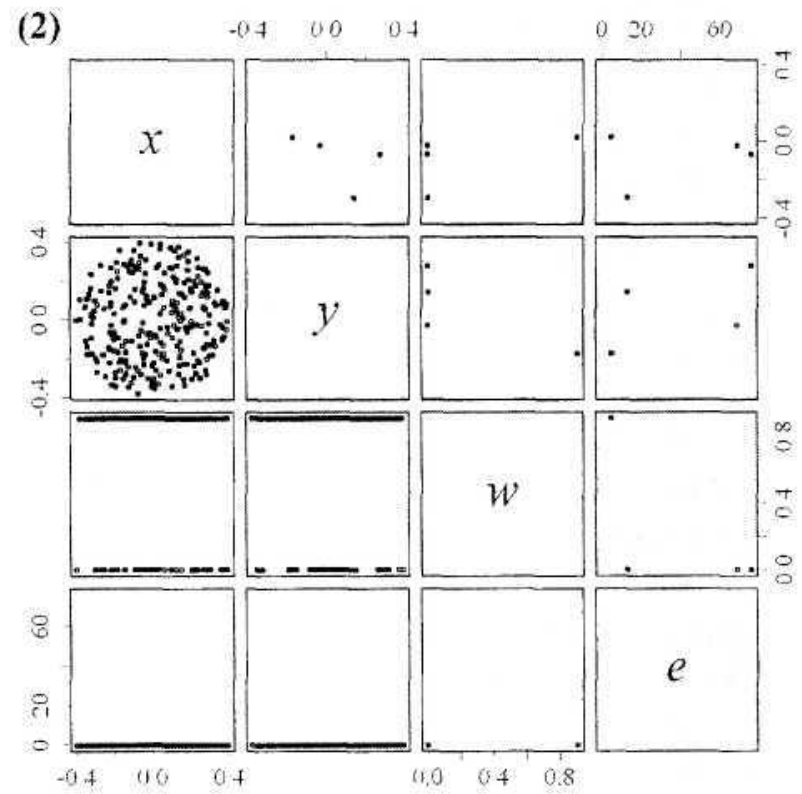

Fig. 10. The inclusion of a second diffractive element may improve significantly filtering and spatial distribution; (1) reference behavior and (2) predicted performance

In Fig. 10, we consider a $12 \mathrm{~mm}$ wide circular aperture, and diffractive elements in a $15 \mathrm{X}$ configuration; with $G=1200$ [lines $/ \mathrm{mm}], m=$ $=+1$, and $\lambda=632.8[\mathrm{~nm}]$. Detection occurs immediately $z=30[\mathrm{~mm}]$ after the sample, case (1) in the figure, or after the diffractive elements, scenario (2). The optical properties of the $10 \mathrm{~mm}$ thick sample are those used throughout this work; $\mu_{\mathrm{a}}=0\left[\mathrm{~mm}^{-1}\right], \mu_{\mathrm{s}}=0.591\left[\mathrm{~mm}^{-1}\right]$, and $g=$ $=0.93$. The filtered distribution retains the circular pattern with a diameter of $800 \mu \mathrm{m}$, but with- out optical noise. Radiation from this coupler may be utilized as input to a BAI. By removing the asymmetry, the system may be employed for imaging and characterization of tissues with ballistic photons.

\section{Conclusions and Future Work}

In the ballistic auto-correlation interferometer (BAI), the arrangement is such that no reference contributions are in play. By developing the theory of the BAI, we predict that for- 
ward-scattered and ballistic information coexists in the interferometric technique. Therefore, we emphasize the importance of implementing an efficient method to separate signal from undesired optical noise. This requirement is especially important for the assessment of biological samples. The latter assertion is a consequence of the forward-directed nature of scattering in tissues.

A non-linear grating-based angular filter may be employed to couple the information of interest to the BAI. The suggested system is conformed by a monochromatic source incident on a ruled grating, positioned at grazing diffraction, followed by a narrow slit. Depending on the angle of incidence of the transmitted radiation, the direction propagation of diffracted radiation is amplified non-linearly. In the experiment, amplification values ranging from $10-20 \mathrm{X}$ are feasible with 1 arc min resolution mounts. More sophisticated equipment may be employed to explore the highly non-linear regime of the coupler.

In transillumination experiments, such as the BAI, the features of angular amplification and decrease in beam extent are exploited to select the information of interest. Furthermore, the proposed system conforms an efficient $\sim 100 \mathrm{X}$ coupling scheme. In contrast to traditional transillumination, the effects of forward-scattered contributions are minimized in the grating-based transmission experiment. In addition, greater exposition times (orders of magnitude) indicate detection of ballistic contributions in the grating-based schemes. Therefore, the proposed method may be suited for coupling selectively the radiation required in the BAI.

Our future research directions will be focused on the realization of a BAI. For this purpose, an important topic of investigation is the plausible implementation of a 2D grating-based coupler as the one discussed in this work. Such system should enable further improvements in filtering efficacy, and hence facilitate the coupling of radiation to the BAI.

This work was carried out during an intership in the "International Institute of Optics and Biophotonics" at Saratov State University. P. Vacas-Jacques gratefully acknowledges the National Science and Technology Council of Mexico (CONACyT) for the financial support given throughout the intership at Saratov State University, grant 172068. The same author thanks the "Centro de Investigaciones en Optica" for partial funding of travel expenses.

This research was performed in the framework of the project "Applications of infrared interferometry for biomedical tomography" (CONACyT 2007-10003-60450), and of the Russian Federation Program for the Development of High School Potential (2009-2010), projects № 2.1.1/4989 and № 2.2.1.1/2950.

\section{References}

1. Patterson M. Wilson B., Wyman D. The propagation of optical radiation in tissue. 1 . Models of radiation transport and their application//Lasers Med. Sci. 1991. Vol.6. P.155-168.

2. Cubeddu R., Pifferi A., Taroni P., Torricelli A., Valentini G. Experimental test of theoretical models for time-resolved reflectance // Med. Phys. 1996. Vol.23. P.1625-1633.

3. Arridge S., Hebden $J$. Optical imaging in medicine: II. Modelling and reconstruction // Phys. Med. Biol. 1997. Vol.42. I.5. P.841-853.

4. Kim A. Transport theory for light propagation in biological tissue // J. Opt. Soc. Amer. A. 2004. Vol.21. I.5. P.820-827.

5. Patterson M., Wilson B., Wyman D. The propagation of optical radiation in tissue. II. Optical properties of tissues and resulting fluence distributions // Lasers Med. Sci. 1991. Vol.6. 1.4. P.379-390.

6. Flock S., Wilson B., Patterson M. Total attenuation coefficients and scattering phase functions of tissues and phantom materials at $633 \mathrm{~nm} / /$ Med. Phys. 1987. Vol.14. I.5. P. $835-841$

7. Cheong W., Prahl S., Welch A. A review of the optical properties of biological tissues // IEEE J. Quantum Elect. 1990 Vol.26(12). P.2166-2185.

8. Лычагов В.В., Лякии Д.В., Модель М.Д., Рябухо В.П. Автокорреляционная пизкокогерентпая интерферометрия рассеивающих и слоистых объектов // Компьютерная оптика. 2007. Т.31, №3. С. 40-51

9. Modell M., Ryahukho V., Lyakin D., Lychagov V., Vitkin E. Itzkan I., Perelman L. Autocorrelation low coherence interferometry // Opt. Commun 2008. Vol.281, №8. P.1991-1996.

10. Vacas-Jacques P., Paez G., Strojnik M. Pass-through photon-based biomedical transillumination // J. Biomed. Opt. 2008. Vol.13. I.4. P.301-307.

11. Vacas-Jacques P., Strojnik M., Puez G. Forward-calculated analytical interferograms in pass-through photon-based biomedical transillumination // J. Opt. Soc. Amer. A 2009. Vol.26. 1.3. P.602-612

12. Vacas-Jacques $P$., Ryabukho V., Strojnik M., Tuchin V., Paez $G$. Theoretical diffractive filter performance for ballistic transillumination // Comp. Opt. / Компьютерная оптика. 2009. T.33, №2. C.129-137.

13. Sun K., Buchman S., Byer R. Grating angle magnification enhanced angular and integrated sensors for LISA applications // J. Phys.: Conf. Ser. 2006. Vol.32. 1.1. P.167-179 
14. Sun $K$., Byer $R$. Grating angle magnification enhanced angular sensor and scanner: United States Patent №20080002197A1, Palo Alto(CA), 2008. Appl.№1 1/820. P.720.

15. Vacas-Jacques P., Ryabukho V., Strojnik M., Tuchin V., Paez $G$. Non-linear grating-based angular filter for ballistic transillumination // Proc. SPIE. Novel Optical Instrumentation for Biomedical Applications / Ed. C. Depeursinge. Bellingham (WA), 2009. Vol.7371.
16. Pogue B., Patterson $M$. Review of tissue simulating phantoms for optical spectroscopy, imaging and dosimetry // J. Biomed. Opt. 2006. Vol.11. 1.4. P.096-102.

17. Fried D., Featherstone J., Darling C., Jones R., Ngaotheppitak $P$., Bühler $C$. Early caries imaging and monitoring with near-infrared light // Dent. Clin. North Amer. 2005. Vol.49. I.4. P. $771-793$. 